\section{Estimating Uncertain Delayed Genetic Regulatory Networks: An Adaptive Filtering Approach}

Wenwu Yu, Student Member, IEEE, Jinhu Lü, Senior Member, IEEE, Guanrong Chen, Fellow, IEEE, Zhisheng Duan, and Qianhe Zhou

\begin{abstract}
Uncertain delayed genetic regulatory networks are investigated from an adaptive filtering approach based on an adaptive synchronization setting. For an unknown regulatory network with time delay and uncertain noise disturbance, several adaptive laws are derived to ensure the stochastic stability of the error states between the unknown network and the estimated model. The novelty lies in the fact that the designed adaptive laws are independent of the unknown system states and parameters, requiring only the output and structure of the underlying network. A representative simulation example is given to verify the effectiveness of the theoretical results.
\end{abstract}

Index Terms-Adaptive filtering, disturbance attenuation, genetic regulatory network (GRN), stochastic stability, system synchronization, time delay.

\section{INTRODUCTION}

Genetic regulatory network (GRN) is a collection of DNA segments in a cell. GRNs interact with each other indirectly, through their RNA and protein expression products, and with other substances in the cell, thereby governing the rates at which genes in the network are transcribed into mRNA. Each mRNA molecule acts for making a specific protein (or, a set of proteins). In some cases, this protein is structural; while in some other cases, this protein is merely an enzyme that catalyzes a certain chemical reaction. Some proteins only serve to activate the other genes, which are the transcription factors binding to the promoter region at the start of other genes turned on by them, initiating the production of another protein, and so on.

Regulatory networks have been extensively studied particularly in biological and biomedical sciences [1]-[10], [29]. Basically, there are two types of genetic network models: the Boolean model [26] and the differential equation model [3]-[5]. In the Boolean model, the activity

Manuscript received February 08, 2008; revised August 23, 2008 and November 11, 2008.Current version published April 08, 2009. This work was supported by the NSFC-HKRGC Joint Research Scheme under Grant N-CityU107/07, the Hong Kong Research Grants Council under the CERG Grant CityU 1117/08E, the National Natural Science Foundation of China under Grants 60821091 and 60772158, the National Basic Research (973) Program of China under Grant 2007CB310805, the Important Direction Project of Knowledge Innovation Program of Chinese Academy of Sciences under Grant KJCX3-SYW-S01, and the Scientific Research Foundation for the Returned Overseas Chinese Scholars, State Education Ministry. Recommended by Associate Editor C.-Y. Su.

W. Yu and G. R. Chen are with the Department of Electronic Engineering, City University of Hong Kong, Hong Kong, China (e-mail: wenwuyu@gmail. com; eegchen@cityu.edu.hk).

J. Lü is with the Institute of Systems Science, Academy of Mathematics and Systems Science, Chinese Academy of Sciences, Beijing 100190, China (e-mail: jhlu@iss.ac.cn).

Z. Duan is with the State Key Lab for Turbulence and Complex Systems, Department of Mechanics and Aerospace Engineering, College of Engineering, Peking University, Beijing 100871, China (e-mail: duanzs@pku.edu.cn).

Q. Zhou is with the Department of Biological Sciences, Columbia University, New York, NY 10027 USA (e-mail: qz2107@ columbia.edu).

Color versions of one or more of the figures in this paper are available online at http://ieeexplore.iee.org.

Digital Object Identifier 10.1109/TAC.2008.2010972 of each gene is expressed by two states, ON or OFF, which are determined by a Boolean function. In the differential equation model, the concentrations of gene products, such as mRNA and proteins, are used as the state variables or their equivalents for modeling.

In this paper, a particular genetic network model is considered, where each transcription factor acts additively to regulate a gene, in such a way that the regulation function sums over all the inputs, therefore is called the SUM logic [5], [21], [22]. In [4]-[6], [9], the regulation function with the SUM logic in a GRN model has been studied in some detail. This paper further investigates the GRN model with time delay and uncertain disturbance.

It is well known that time delay is ubiquitous in biological, physical, chemical, and electrical dynamical systems. In biological systems, particularly in GRNs, time delays are inevitable due to the slow process of transcription, translation, and translocation processes therein. It has been observed from both numerical and laboratory experiments that time delays can derail the stability of the system thereby causing sustained oscillations, bifurcations, or even chaos [11], [14], [15]. More seriously speaking, mathematical models without addressing time delays may actually provide wrong predictions of the mRNA and protein concentrations.

In modeling GRNs, on the other hand, molecular noise has been shown to play an important role in biological functions since noise is unavoidable in reactions of transcription, translation, and translocation processes, and also due to external fluctuations. There exist some studies on GRNs with random disturbances in the literature [7], [8], where the states of the system are fully known. However, it has been pointed out that in practice, some concentrations of products, such as mRNA, protein, and network parameters may not be fully known, hence estimation becomes impossible based on the previous results.

Moreover, in a real GRN it is still not completely understood today as how the genes are expressed in the right time and right place, with the right amount, throughout the development of the organism. Studying living organisms is a fairly complex process, which requires significant work on observing, collecting, and analyzing data. Sometimes, it is even impossible for biologists to get all the information due to various technical difficulties. Thus, the study of system biology comes in place, where it attempts to estimate the missing information from the available data. This gives rise to the following filtering problem: given a delayed genetic regulatory network with unknown parameters and random disturbance, how to estimate the unknown products and parameters by using the observed data from the network?

Clearly, a combination of time delay, noise disturbance, and unknown parameters and system states makes the study of uncertain GRN models very difficult.

In this paper, the adaptive filtering technique is employed to estimate the unknown parametric information such as the mRNA degradation rate, transcription rate, protein, and so on, from the available mRNA data. More precisely, the adaptive filtering problem for unknown delayed GRNs with disturbance attenuation is investigated, based on a system synchronization setting. The adaptive filtering of unknown dynamical systems without random disturbance has already been studied in [12], [13], [16]-[20], [30]. The synchronization of nonlinear systems with random disturbance, on the other hand, has also been investigated, in [15], [24], [25], where the noise was assumed to exist only in the response system and it vanishes on the synchronization manifold. In this paper, a more realistic situation where noise exists in the master system is considered; that is, an uncertain GRN model is considered, where noise does not vanish on the synchronization manifold. Furthermore, note that in the aforementioned references, i.e., [12], [13], [15]-[20], [24], [25], [31], all the states of the networks are supposed to be known, 
which certainly is rare in real biological systems. In contrast, in this paper, some states and system parameters are allowed to be unknown in the concerned delayed GRN model with random disturbances.

The rest of this paper is organized as follows: First, in Section II, model formulation and preliminaries of the GRN model are briefly outlined, and a delayed GRN model with unknown parameter and random disturbance is described. Then, an adaptive filter is designed to estimate the unknown states and parameters of the model in Section III. To that end, in Section IV, a numerical example is shown to demonstrate the effectiveness of the proposed adaptive filtering approach. Finally, conclusions are drawn in Section V.

\section{MODEL FORMULATION AND PRELIMINARIES}

\section{A. Original GRN Model}

Consider the delayed GRN model [4]-[6]

$$
\begin{aligned}
& \frac{d m_{i}(t)}{d t}=-a_{i} m_{i}(t)+G_{i}\left(p_{1}(t-\tau), p_{2}(t-\tau), \ldots,\right.\left.p_{n}(t-\tau)\right), \\
& \frac{d p_{i}(t)}{d t}=-c_{i} p_{i}(t)+d_{i} m_{i}(t-\tau)
\end{aligned}
$$

where $m_{i}(t), p_{i}(t) \in R$ are concentrations of mRNA and protein of the $i$ th node at time $t, a_{i}>0$ and $c_{i}>0$ are the degradation rates of the mRNA and protein, respectively, $d_{i}$ is the translation rate, $\tau$ is a time delay, and the function $G_{i}$ represents the feedback regulation of the protein on the transcription of the $i$ th node, which is generally a nonlinear monotonically increasing function [3]-[10], for all $i=1,2, \cdots, n$.

The gene activities are being controlled in a cell, and the gene regulation function $G_{i}$ plays a key role in determining the dynamical behavior of the network. Generally, $G_{i}$ can be very complex. In [5], [6], it is assumed that each transcription factor acts additively to regulate the $i$ th gene, and the regulatory function is in the form of $G_{i}=$ $\sum_{j=1}^{n} G_{i j}\left(p_{j}(t)\right)$, which is also called the SUM logic [21], [22]. Moreover, each $G_{i j}$ is assumed to be a monotonic function in the so-called Hill form [8], namely, if the transcription factor $j$ is an activator of gene $i$, then

$$
G_{i j}\left(p_{j}(t)\right)=\alpha_{i j} \frac{\left(p_{j}(t) / \beta_{j}\right)^{H_{j}}}{1+\left(p_{j}(t) / \beta_{j}\right)^{H_{j}}}
$$

if transcription factor $j$ is a repressor of gene $i$, then

$$
G_{i j}\left(p_{j}(t)\right)=\alpha_{i j} \frac{1}{1+\left(p_{j}(t) / \beta_{j}\right)^{H_{j}}}
$$

where $H_{j}$ are called the Hill coefficients, $\beta_{j}$ are positive constants, and $\alpha_{i j}$ are the dimensionless transcriptional rate of the transcription factor $j$ to $i$.

Thus, system (1) can be rewritten as

$$
\begin{aligned}
\frac{d m_{i}(t)}{d t} & =-a_{i} m_{i}(t)+\sum_{j=1}^{n} w_{i j} f_{j}\left(p_{j}(t-\tau)\right)+L_{i}, \\
\frac{d p_{i}(t)}{d t} & =-c_{i} p_{i}(t)+d_{i} m_{i}(t-\tau)
\end{aligned}
$$

where $f_{j}(x)=\left(x / \beta_{j}\right)^{H_{j}} /\left(1+\left(x / \beta_{j}\right)^{H_{j}}\right)$, and $L_{i}=\sum_{j \in I_{i}} \alpha_{i j}$ in which $I_{i}$ is the set of all the repressors of gene $i$, with $W=\left(w_{i j}\right) \in$ $R^{n \times n}$ defined as

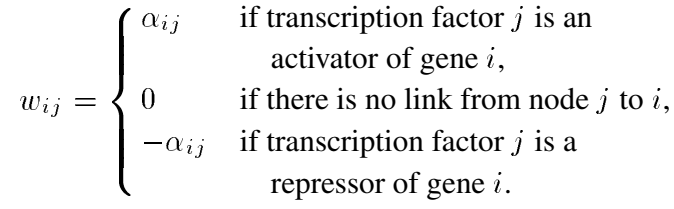

System (4) can be further written in a compact matrix form as follows:

$$
\begin{aligned}
\frac{d m(t)}{d t} & =-A m(t)+W f(p(t-\tau))+L, \\
\frac{d p(t)}{d t} & =-C p(t)+D m(t-\tau)
\end{aligned}
$$

where $m(t)=\left[m_{1}(t), m_{2}(t), \ldots, m_{n}(t)\right]^{T}, p(t)=$ $\left[p_{1}(t), p_{2}(t), \ldots, p_{n}(t)\right]^{T}, \quad f(p(t-\tau))=\left[f_{1}\left(p_{1}(t-\right.\right.$ $\left.\tau)), f_{2}\left(p_{2}(t-\tau)\right), \ldots, f_{n}\left(p_{n}(t-\tau)\right)\right]^{T}, m(t-\tau)=$ $\left[m_{1}(t-\tau), m_{2}(t-\tau), \ldots, m_{n}(t-\tau)\right]^{T}, L=\left(L_{1}, L_{2}, \ldots, L_{n}\right)^{T}$, $A=\operatorname{diag}\left\{a_{1}, a_{2}, \ldots, a_{n}\right\}, C=\operatorname{diag}\left\{c_{1}, c_{2}, \ldots, c_{n}\right\}$, $D=\operatorname{diag}\left\{d_{1}, d_{2}, \ldots, d_{n}\right\}$, and $W=\left(w_{i j}\right)_{n \times n}$.

\section{B. The Uncertain GRN Model}

In this paper, an uncertain GRN model with random disturbance is considered

$$
\begin{aligned}
d m(t) & =[-A m(t)+W f(p(t-\tau))+L] d t+v(t) d \nu, \\
d p(t) & =[-C p(t)+D m(t-\tau)] d t+\sigma(t) d \omega, \\
y & =C_{0} x(t)
\end{aligned}
$$

where $y$ is the output, $x(t)=\left[m^{T}(t), p^{T}(t)\right]^{T}, C_{0}=\left(I_{n} 0_{n}\right)$, with $I_{n}$ and $0_{n}$ denoting the $n$-dimension identity and zero matrices, respectively, $v(t)$ and $\sigma(t)$ are external noise intensity functions, and $\omega(t)$ and $\nu(t)$ are two independent one-dimensional Brownian motions satisfying the mathematical expectations $\mathbf{E}\{d \omega(t)\}=0, \mathbf{E}\{d \nu(t)\}=0$, $\mathbf{E}\left\{d \omega(t)^{2}\right\}=1$ and $\mathbf{E}\left\{d \nu(t)^{2}\right\}=1$.

The initial conditions of (6) are given by $x_{i}(t)=\phi_{i}(t) \epsilon$ $\mathcal{C}([-\tau, 0], R)$, which is the set of real-valued continuous functions on $[-\tau, 0]$ for some $\tau>0$.

Sometimes, the concentrations of mRNA can be obtained. However, the regulation process in mRNA, i.e, the degradation rates $a_{i}$, transcriptional factors $w_{i j}$, repressor factors $L_{i}$, and concentrations of protein $p(t)$, are likely unknown and not available for direct measuring. Thus, assume that in system (6), $A, W, L$ are uncertain matrices and $p(t)$ is unknown but the output $y=m(t)$ can be observed. The objective then is to estimate the concentrations of protein.

To realize this goal, an adaptive filtering approach is adopted below. In general, a nonlinear filtering is constructed in the following form:

$$
\begin{aligned}
\frac{d \widetilde{m}(t)}{d t}= & -\widetilde{A}(t) \widetilde{m}(t)+\widetilde{W}(t) f(\widetilde{p}(t-\tau)) \\
& +\widetilde{L}(t)-K(t)(\widetilde{m}(t)-y(t)), \\
\frac{d \widetilde{p}(t)}{d t}= & -C \widetilde{p}(t)+D \widetilde{m}(t-\tau)
\end{aligned}
$$

where $\tilde{m}(t)=\left[\tilde{m}_{1}(t), \tilde{m}_{2}(t), \ldots, \widetilde{m}_{n}(t)\right]^{T}, \tilde{p}(t)=$ $\left[\widetilde{p}_{1}(t), \widetilde{p}_{2}(t), \ldots, \widetilde{p}_{n}(t)\right]^{T}, K(t)=\operatorname{diag}\left\{k_{1}(t), k_{2}(t), \ldots, k_{n}(t)\right\}$ is the adaptive feedback matrix, $\widetilde{W}(t)=\left(\widetilde{w}_{i j}(t)\right)_{n \times n}, \widetilde{A}(t)=$ $\operatorname{diag}\left\{\widetilde{a}_{1}(t), \widetilde{a}_{2}(t), \ldots, \widetilde{a}_{n}(t)\right\}$, and $\widetilde{L}(t)=\left[\widetilde{l}_{1}(t), \widetilde{l}_{1}(t), \ldots, \widetilde{l}_{n}(t)\right]^{T}$ are matrix- and vector-valued functions of time $t$. 
Note that systems (6) and (7) can be considered as the master and slave systems in a typical synchronization setting, as discussed in [13], [14], [16].

Now, subtracting (6) from (7) yields the following error dynamical system:

$$
\begin{aligned}
d e_{m}(t)= & {[-\widetilde{A}(t) \widetilde{m}(t)+\widetilde{W}(t) f(\widetilde{p}(t-\tau))+\widetilde{L}(t)+A m(t)} \\
& \left.-W f(p(t-\tau))-L-K e_{m}(t)\right] d t-v(t) d \nu \\
d e_{p}(t)= & {\left[-C e_{p}(t)+D e_{m}(t-\tau)\right] d t-\sigma(t) d \omega }
\end{aligned}
$$

where $e_{m}(t)=\widetilde{m}(t)-m(t), e_{p}(t)=\widetilde{p}(t)-p(t)$, $e_{m}(t)=\left[e_{m 1}(t), e_{m 2}(t), \ldots, e_{m n}(t)\right]^{T}, \quad e_{p}(t)=$ $\left[e_{p 1}(t), e_{p 2}(t), \ldots, e_{p n}(t)\right]^{T}$.

Note also that in system (4), the function $f_{i}: R \rightarrow R$ is monotonically increasing and satisfies the Lipschitz condition

$$
\left|f_{i}(u)-f_{i}(v)\right| \leq h_{i}|u-v| \quad \forall u, v \in R
$$

with the Lipschitz constants $h_{i}>0$ for all $i=1,2, \ldots, n$.

Next, some useful concept and preliminaries are introduced.

Definition 1 [8]: The two networks (6) and (7) are said to be stochastically synchronous with disturbance attenuation $\gamma>0$, if

i) network (8) with $v(t)=0$ and $\sigma(t)=0$ is asymptotically stable;

ii) with zero initial conditions, there exists a scalar $\gamma>0$ such that

$$
\begin{aligned}
& \mathbf{E} \int_{0}^{\infty}\left(\left\|e_{m}(s)\right\|^{2}+\left\|e_{p}(s)\right\|^{2}\right) d s \\
& \leq \gamma^{2} \int_{0}^{\infty}\left(\|v(s)\|^{2}+\|\sigma(s)\|^{2}\right) d s
\end{aligned}
$$

for all nonzero $v, \sigma \in L_{2}[0, \infty)$.

Lemma 1: For any vectors $x, y \in R^{n}$ and positive definite matrix $G \in R^{n \times n}$, the following matrix inequality holds:

$$
2 x^{T} y \leq x^{T} G x+y^{T} G^{-1} y .
$$

\section{ADAPTIVE FILTER DESIGN}

In this section, some adaptive laws are designed for estimating the unknown terms of $K(t), \widetilde{A}(t), \widetilde{W}(t)$, and $\widetilde{L}(t)$, so as to achieve the asymptotical stability of the error dynamical system (8) with disturbance attenuation.

First, some simple sufficient conditions are given, as follows.

Theorem 1: The two networks (6) and (7) are stochastically synchronous with disturbance attenuation $\gamma>0$, if

$$
\begin{aligned}
\dot{\widetilde{a}}_{i} & =q_{i} e_{m i} \widetilde{m}_{i}(t), \quad i=1,2, \ldots, n \\
\dot{\widetilde{w}}_{i j} & =-r_{i j} e_{m i} f_{j}(\widetilde{p}(t-\tau)), \quad i, j=1,2, \ldots, n \\
\dot{\widetilde{l}}_{i} & =-u_{i} e_{m i}, \quad i=1,2, \ldots, n \\
\dot{k}_{i} & =\beta_{i} e_{m i}^{2}
\end{aligned}
$$

and

$$
\gamma>\frac{1}{\sqrt{2(c-\mu)}}
$$

where $q_{i}, r_{i j}, u_{i}$, and $\beta_{i}$ are positive constants, $c=\min _{1 \leq i \leq n}\left\{c_{i}\right\}$, and $\mu$ is a positive constant satisfying $\mu<c$.

Proof: Consider the Lyapunov functional

$$
V(t)=\frac{1}{2}\left[e_{m}^{T}(t) e_{m}(t)+e_{p}^{T}(t) e_{p}(t)\right]+\sum_{i=1}^{n} \frac{1}{2 q_{i}}\left(\widetilde{a}_{i}-a_{i}\right)^{2}
$$

$$
\begin{aligned}
& +\sum_{i=1}^{n} \sum_{j=1}^{n} \frac{1}{2 r_{i j}}\left(\widetilde{w}_{i j}-w_{i j}\right)^{2} \\
& +\sum_{i=1}^{n} \frac{1}{2 u_{i}}\left(\widetilde{l}_{i}-l_{i}\right)^{2}+\sum_{i=1}^{n} \frac{1}{2 \beta_{i}}\left(k_{i}-\mathbf{k}\right)^{2} \\
& +\int_{t-\tau}^{t}\left[\delta_{1} e_{m}^{T}(s) e_{m}(s)+\delta_{2} e_{p}^{T}(s) e_{p}(s)\right] d s
\end{aligned}
$$

where $\mathbf{k}, \delta_{1}$, and $\delta_{2}$ are positive constants to be determined.

From the Itô formula [23], one obtains the following stochastic differential:

$$
d V(t)=\mathcal{L} V(t) d t-e_{m}^{T}(t) v(t) d \nu-e_{p}^{T}(t) \sigma(t) d \omega
$$

The weak infinitesimal operator $\mathcal{L}$ of the stochastic process is given by

$$
\begin{aligned}
& \mathcal{L} V(t)=e_{m}^{T}(t)[-\widetilde{A}(t) \widetilde{m}(t)+\widetilde{W}(t) f(\widetilde{p}(t-\tau))+\widetilde{L}(t) \\
& \left.+A m(t)-W f(p(t-\tau))-L-K e_{m}(t)\right] \\
& +e_{p}^{T}(t)\left[-C e_{p}(t)+D e_{m}(t-\tau)\right] \\
& +\sum_{i=1}^{n}\left(\widetilde{a}_{i}-a_{i}\right) e_{m i} \tilde{m}_{i}(t) \\
& -\sum_{i=1}^{n} \sum_{j=1}^{n}\left(\widetilde{w}_{i j}-w_{i j}\right) e_{m i} f_{j}\left(\widetilde{p}_{j}(t-\tau)\right) \\
& -\sum_{i=1}^{n}\left(\tilde{l_{i}}-l_{i}\right) e_{m i}+\sum_{i=1}^{n}\left(k_{i}-\mathbf{k}\right) e_{m i}^{2} \\
& +\delta_{1}\left[e_{m}^{T}(t) e_{m}(t)-e_{m}^{T}(t-\tau) e_{m}(t-\tau)\right] \\
& +\delta_{2}\left[e_{p}^{T}(t) e_{p}(t)-e_{p}^{T}(t-\tau) e_{p}(t-\tau)\right] \\
& +\frac{1}{2} v^{T}(t) v(t)+\frac{1}{2} \sigma^{T}(t) \sigma(t) \\
& =e_{m}^{T}(t)[-(\tilde{A}(t)-A) \tilde{m}(t) \\
& +(\widetilde{W}(t)-W) f(\widetilde{p}(t-\tau)) \\
& -\left(A+\mathbf{k} I_{n}-\delta_{1} I_{n}\right) e_{m}(t)+(\widetilde{L}(t)-L) \\
& +W(f(\widetilde{p}(t-\tau))-f(p(t-\tau)))] \\
& -e_{p}^{T}(t)\left(C-\delta_{2} I_{n}\right) e_{p}(t)+e_{p}^{T} D e_{m}(t-\tau) \\
& +\sum_{i=1}^{n}\left(\widetilde{a}_{i}-a_{i}\right) e_{m i} \tilde{m}_{i}(t) \\
& -\sum_{i=1}^{n} \sum_{j=1}^{n}\left(\widetilde{w}_{i j}-w_{i j}\right) e_{m i} f_{j}\left(\widetilde{p}_{j}(t-\tau)\right) \\
& -\sum_{i=1}^{n}\left(\widetilde{l_{i}}-l_{i}\right) e_{m i}-\delta_{1} e_{m}^{T}(t-\tau) e_{m}(t-\tau) \\
& -\delta_{2} e_{p}^{T}(t-\tau) e_{p}(t-\tau)+\frac{1}{2} v^{T}(t) v(t) \\
& +\frac{1}{2} \sigma^{T}(t) \sigma(t) \\
& \leq-e_{m}^{T}(t)\left(A+\mathbf{k} I_{n}-\delta_{1} I_{n}\right) e_{m}(t) \\
& +e_{m}^{T}(t) W[f(\widetilde{p}(t-\tau))-f(p(t-\tau))] \\
& -e_{p}^{T}(t)\left(C-\delta_{2} I_{n}\right) e_{p}(t)+e_{p}^{T} D e_{m}(t-\tau) \\
& -\delta_{1} e_{m}^{T}(t-\tau) e_{m}(t-\tau)-\delta_{2} e_{p}^{T}(t-\tau) e_{p}(t-\tau) \\
& +\frac{1}{2} v^{T}(t) v(t)+\frac{1}{2} \sigma^{T}(t) \sigma(t) \text {. }
\end{aligned}
$$


According to the Lipschitz condition (9) and Lemma 1, one has

$$
\begin{array}{r}
e_{m}^{T}(t) W[f(\widetilde{p}(t-\tau))-f(p(t-\tau))] \leq \frac{1}{2 \eta} e_{m}^{T}(t) W W^{T} e_{m}(t) \\
+\frac{\eta h^{2}}{2} e_{p}^{T}(t-\tau) e_{p}(t-\tau)
\end{array}
$$

and

$$
e_{p}^{T} D e_{m}(t-\tau) \leq \frac{\varepsilon}{2} e_{p}^{T}(t) D D^{T} e_{p}(t)+\frac{1}{2 \varepsilon} e_{m}^{T}(t-\tau) e_{m}(t-\tau)
$$

where $h=\max _{1 \leq i \leq n}\left\{h_{i}\right\}, \eta$ and $\varepsilon$ are positive constants.

Substituting (18) and (19) into (17) yields

$$
\begin{aligned}
\mathcal{L} V(t) \leq & -e_{m}^{T}(t)\left(A+\mathbf{k} I_{n}-\delta_{1} I_{n}-\frac{1}{2 \eta} W W^{T}\right) e_{m}(t) \\
& -e_{p}^{T}(t)\left(C-\delta_{2} I_{n}-\frac{\varepsilon}{2} D D^{T}\right) e_{p}(t) \\
& +\left(\frac{1}{2 \varepsilon}-\delta_{1}\right) e_{m}^{T}(t-\tau) e_{m}(t-\tau) \\
& +\left(\frac{\eta}{2} h^{2}-\delta_{2}\right) e_{p}^{T}(t-\tau) e_{p}(t-\tau) \\
& +\frac{1}{2} v^{T}(t) v(t)+\frac{1}{2} \sigma^{T}(t) \sigma(t)
\end{aligned}
$$

If one chooses $\delta_{1}=1 / 2 \varepsilon$ and $\delta_{2}=(\eta / 2) h^{2}$, then

$$
\begin{aligned}
\mathcal{L} V(t) \leq & -e_{m}^{T}(t)\left(A+\mathbf{k} I_{n}-\frac{1}{2 \varepsilon} I_{n}-\frac{1}{2 \eta} W W^{T}\right) e_{m}(t) \\
& -e_{p}^{T}(t)\left(C-\frac{\eta}{2} h^{2} I_{n}-\frac{\varepsilon}{2} D D^{T}\right) e_{p}(t) \\
& +\frac{1}{2} v^{T}(t) v(t)+\frac{1}{2} \sigma^{T}(t) \sigma(t) \\
\leq & -\left(a+\mathbf{k}-\frac{1}{2 \varepsilon}-\frac{1}{2 \eta} \lambda_{\max }\left(W W^{T}\right)\right) e_{m}^{T}(t) e_{m}(t) \\
& -\left(c-\frac{\eta}{2} h^{2}-\frac{\varepsilon}{2} \lambda_{\max }\left(D D^{T}\right)\right) e_{p}^{T}(t) e_{p}(t) \\
& +\frac{1}{2} v^{T}(t) v(t)+\frac{1}{2} \sigma^{T}(t) \sigma(t)
\end{aligned}
$$

where $a=\min _{1 \leq i \leq n}\left\{a_{i}\right\}, c=\min _{1 \leq i \leq n}\left\{c_{i}\right\}$, and $\lambda_{\max }(M)$ denotes the maximal eigenvalue of the symmetric matrix $M$.

For $\gamma>0$, set

$$
\begin{aligned}
J(t)=\mathbf{E} \int_{0}^{t}\left[e_{m}^{T}(s) e_{m}(s)\right. & +e_{p}^{T}(s) e_{p}(s) \\
& \left.-\gamma^{2} v^{T}(s) v(s)-\gamma^{2} \sigma^{T}(s) \sigma(s)\right] d s .
\end{aligned}
$$

Then, from (15), (16), (22) and under zero initial conditions, for $\rho>0$ one has

$$
\begin{array}{r}
J(t) \leq \mathbf{E} \int_{0}^{t}\left[e_{m}^{T}(s) e_{m}(s)+e_{p}^{T}(s) e_{p}(s)-\gamma^{2} v^{T}(s) v(s)\right. \\
\left.-\gamma^{2} \sigma^{T}(s) \sigma(s)+\rho \mathcal{L} V(s)\right] d s .
\end{array}
$$

Let $\rho=2 \gamma^{2}$. Then, from (21) it follows that:

$$
\begin{aligned}
J(t) \leq \mathbf{E} \int_{0}^{t} 2 \gamma^{2}[- & \left(a+\mathbf{k}-\frac{1}{2 \varepsilon}\right. \\
& \left.-\frac{1}{2 \eta} \lambda_{\max }\left(W W^{T}\right)-\frac{1}{2 \gamma^{2}}\right) e_{m}^{T}(s) e_{m}(s) \\
- & \left(c-\frac{\eta}{2} h^{2}-\frac{\varepsilon}{2} \lambda_{\max }\left(D D^{T}\right)-\frac{1}{2 \gamma^{2}}\right) \\
& \left.\times e_{p}^{T}(s) e_{p}(s)\right] d s
\end{aligned}
$$

Choose sufficiently small values $\eta$ and $\varepsilon$, so that $c-(\eta / 2) h^{2}-$ $(\varepsilon / 2) \lambda_{\max }\left(D D^{T}\right)>0$, and let $\mu=(\eta / 2) h^{2}+(\varepsilon / 2) \lambda_{\max }\left(D D^{T}\right)$, $\mathbf{k}=-a+(1 / 2 \varepsilon)+(1 / 2 \eta) \lambda_{\max }\left(W W^{T}\right)+\left(1 / 2 \gamma^{2}\right)+1$. It is then easy to verfity that the condition i) in Definition 1 is satisfied.

Next, it is to find the disturbance attenuation $\gamma$, so that condition ii) in Definition 1 is also satisfied.

By (23), one has

$$
\begin{aligned}
J(t) \leq \mathbf{E} \int_{0}^{t} 2 \gamma^{2}\left[-e_{m}^{T}(s)\right. & e_{m}(s) \\
& \left.-\left(c-\mu-\frac{1}{2 \gamma^{2}}\right) e_{p}^{T}(s) e_{p}(s)\right] d s .
\end{aligned}
$$

From (14) and (22), it follows that $J(t)<0$. Consequently

$$
\begin{aligned}
\mathbf{E} \int_{0}^{t}\left[e_{m}^{T}(s) e_{m}(s)+\right. & \left.e_{p}^{T}(s) e_{p}(s)\right] d s \\
& \leq \gamma^{2} \mathbf{E} \int_{0}^{t}\left[v^{T}(s) v(s)+\sigma^{T}(s) \sigma(s)\right] d s .
\end{aligned}
$$

Therefore, with the designed adaptive laws (10)-(13), the condition ii) in Definition 1 is satisfied. This completes the proof of the theorem.

Remark 1: Under the adaptive laws (10)-(13), one has $\gamma>(1 / \sqrt{2(c-\mu)})$. By choosing a sufficiently small value $\mu$, one can obtain the low bound of $\gamma=1 / \sqrt{2 c}$. Thus, by adaptive laws (10)-(13), the network synchronous error dynamical system (8) is stochastically stable about zero, with disturbance attenuation $\gamma=1 / \sqrt{2 c}$. In other words, the adaptive filter (7) has been successfully designed.

Remark 2: In [15], [24], [25], the synchronization problem of nonlinear systems with stochastic disturbance was investigated. However, the noise intensity function therein is $\sigma(e(t), e(t-\tau))$ in the response system, where $e$ is the error state, which can vanish on the synchronization manifold $e(t)=0$. It is not realistic to assume that noise exists in the response rather than in the master system. In this paper, a more general and more practical case is considered, thereby it is assumed that noise exists in the master system, i.e., in the GRN (6), and it may not vanish on the synchronization manifold.

Remark 3: In [27], [28], robust Kalman filter is used for discrete linear time-varying uncertain systems with noise and possibly missing measurements, while in this paper the uncertain system parameters are assumed fully unknown and the GRN is nonlinear. Hence, the approach under consideration here is more general.

\section{A Simulation EXAMPle}

In this section, a representative numerical example is given to demonstrate the effectiveness of the proposed synchronization-based adaptive filtering technique.

The dynamics of the repressilator has been theoretically predicted and experimentally verified on Escherichia coli [3]. Three repressorprotein concentrations $p_{i}$, and their mRNA concentrations $m_{i}$ (where $i$ is $l a c l$, tet $R$ or $c l$ ) were used as continuous dynamical variables. The repressilator is a cyclic negative-feedback loop composing of three genes and their corresponding promoters. The kinetics of the system are determined by six coupled first-order differential equations, as follows:

$$
\begin{aligned}
\frac{d m_{i}(t)}{d t} & =-m_{i}(t)+\frac{\alpha}{1+p_{j}^{n}(t)}+L_{i}, \\
\frac{d p_{i}(t)}{d t} & =-c_{i} p_{i}(t)+d_{i} m_{i}(t)
\end{aligned}
$$

where $i=l a c l, t e t R, c l ; j=c l, l a c l, t e t R$, and $n$ is a Hill coefficient. 


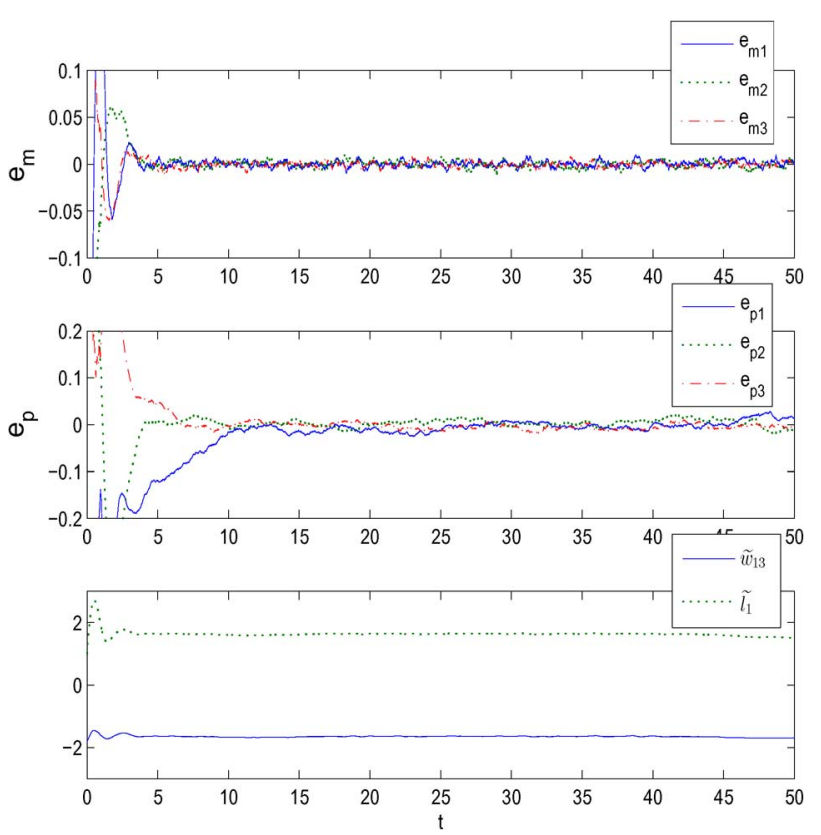

Fig. 1. Error states $e_{m}$ and $e_{p}$, and adaptive laws $\widetilde{w}_{13}$ and $\widetilde{l}_{1}$.

Taking into account the transcriptional time delay and stochastic disturbance, the following unknown GRN model is considered [4], [5], [27]:

$$
\begin{aligned}
d m(t) & =[-A m(t)+W f(p(t-\tau))+L] d t+v(t) d \nu, \\
d p(t) & =[-C p(t)+D m(t-\tau)] d t+\sigma(t) d \omega, \\
y & =m(t)
\end{aligned}
$$

where $A=\left(\begin{array}{ccc}0.4 & 0 & 0 \\ 0 & 0.36 & 0 \\ 0 & 0 & 0.48\end{array}\right), C=\left(\begin{array}{ccc}0.2 & 0 & 0 \\ 0 & 0.5 & 0 \\ 0 & 0 & 0.6\end{array}\right), W=$ $1.5\left(\begin{array}{ccc}0 & 0 & -1 \\ -1 & 0 & 0 \\ 0 & -1 & 0\end{array}\right), D=2\left(\begin{array}{lll}1 & 0 & 0 \\ 0 & 1 & 0 \\ 0 & 0 & 1\end{array}\right), L=\left(\begin{array}{lll}1.5 & 1.5 & 1.5\end{array}\right)^{T}$, $v(t)=0.1 e^{-0.05 t}, \sigma(t)=e^{-0.1 t}, f(z)=z^{2} /\left(1+z^{2}\right), \tau=1$.

Assume $w_{13}=-1.5$ and $l_{1}=1.5$ are unknown, and the adaptive filter (7) and (10)-(13) is designed. The error states $e_{m}$ and $e_{p}$ in (8), and the adaptive laws $\widetilde{w}_{13}$ and $\widetilde{l}_{1}$ in (11) and (12), respectively, are shown in Fig. 1, where $r_{13}=u_{1}=10$.

From Fig. 1, it is clear that the adaptive filter (7) and (10)-(13) works very effectively. It can also be seen that parameters $w_{13}=-1.5$ and $l_{1}=1.5$ could be approximately estimated by $\widetilde{w}_{13}$ and $\widetilde{l}_{1}$, as expected.

Remark 4: Somewhat related, in [13], [16] system parameter identification is carried out using time-series data, where a linear independence condition is derived.

\section{Conclusion}

In this paper, an adaptive filtering approach has been developed, based on the adaptive synchronization setting, for estimating unknown delayed genetic regulatory networks with noise disturbance. Several adaptive laws have been derived to ensure the stochastic stability of the synchronous errors between the unknown delayed genetic regulatory network and its estimated model with noise disturbance. The designed adaptive laws are independent of the unknown system states and parameters, and only the system output and structure are assumed. In addition to assuming the system parameters to be unknown, noise is allowed to exist in the genetic regulatory network, which may not vanish on the synchronization manifold. The success of the proposed adaptive filtering technique shows that it is a powerful and promising approach therefore should be further explored for more applications in the future.

\section{ACKNOWLEDGMENT}

The authors would like to thank Dr. Z. Wang, Dr. J. Cao, and Dr. F. Ren for some inspiring discussions and helpful suggestions.

\section{REFERENCES}

[1] Z. Wang, H. Gao, J. Cao, and X. Liu, "On delayed genetic regulatory networks with polytopic uncertainties: Robust stability analysis," IEEE Trans. NanoBiosci., vol. 7, no. 2, pp. 154-163, Jun. 2008.

[2] G. Wei, Z. Wang, H. Shu, K. Fraser, and X. Liu, "Robust filtering for gene expression time series data with variance constraints," Int. J. Comput. Math., vol. 84, no. 5, pp. 619-633, May 2007.

[3] M. B. Elowitz and S. Leibler, "A synthetic oscillatory network of transcriptional regulators," Nature, vol. 403, pp. 335-338, 2000.

[4] L. Chen and K. Aihara, "Stability of genetic regulatory networks with time delay," IEEE Trans. Circuits Syst. I, vol. 49, no. 5, pp. 602-608, May 2002.

[5] C. Li, L. Chen, and K. Aihara, "Stability of genetic networks with SUM regulatory logic: Lur'e System and LMI approach," IEEE Trans. Circuits Syst. I, vol. 53, no. 11, pp. 2451-2458, Nov. 2006.

[6] F. Ren and J. Cao, "Asymptotic and robust stability of genetic regulatory networks with time-varying delays," Neurocomputing, vol. 71, pp. 834-842, 2008.

[7] C. Li, L. Chen, and K. Aihara, "Stochastic stability of genetic networks with disturbance attenuation," IEEE Trans. Circuits Syst. II, vol. 54, no. 10, pp. 892-896, Oct. 2007.

[8] C. Li, L. Chen, and K. Aihara, "Stochastic synchronization of genetic oscillator networks," BMC Syst. Biol. vol. 1, no. 6, Jan. 2007 [Online]. Available: http://arxiv.org/abs/q-bio/0702025

[9] J. Cao and F. Ren, "Exponential stability of discrete-time genetic regulatory networks with delays," IEEE Trans. Neural Networks, vol. 19, no. 3, pp. 520-523, May 2008.

[10] B. Xu and Y. Tao, "External noise and feedback regulation: Steadystate statistics of auto-regulatory genetic network," J. Theoret. Biol., vol. 243, pp. 214-221, 2006.

[11] W. Yu, J. Cao, and G. Chen, "Stability and Hopf bifurcation of a general delayed recurrent neural network," IEEE Trans. Neural Networks, vol. 19, no. 5, pp. 845-854, Sep. 2008.

[12] W. Yu, J. Cao, and G. Chen, "Robust adaptive control of unknown modified Cohen-Grossberg neural networks with delay," IEEE Trans. Circuits Syst. II, vol. 54, no. 6, pp. 502-506, Jun. 2007.

[13] W. Yu, G. Chen, J. Cao, J. Lü, and U. Parlitz, "Parameter identification of dynamical systems from time series," Phys. Rev. E, vol. 75, no. 6, p. 067201, 2007.

[14] W. Yu, J. Cao, K. W. Wong, and J. Lü, "New communication schemes based on adaptive synchronization," Chaos, vol. 17, p. 033114, 2007.

[15] W. Yu and J. Cao, "Synchronization control of stochastic delayed neural networks," Physica A, vol. 373, pp. 252-260, 2007.

[16] W. Yu and J. Cao, "Adaptive Q-S (lag, anticipated, and complete) timevarying synchronization and parameters identification of uncertain delayed neural networks," Chaos, vol. 16, p. 023119, 2006.

[17] D. Huang, "Adaptive-feedback control algorithm," Phys. Rev. E, vol. 73, p. 066204, 2006.

[18] D. Huang, "Stabilizing near-nonhyperbolic chaotic systems with applications," Phys. Rev. Lett., vol. 93, p. 214101, 2004.

[19] J. Zhou, T. Chen, and L. Xiang, "Adaptive synchronization of coupled chaotic delayed systems based on parameter identification and its applications," Int. J. Bifur. Chaos, vol. 16, no. 10, pp. 2923-2933, 2006.

[20] J. Cao and J. Lu, "Adaptive synchronization of neural networks with or without time-varying delays," Chaos, vol. 16, p. 013133, 2006.

[21] C. H. Yuh, H. Bolouri, and E. H. Davidson, "Genomic cis-regulatory logic: Experimental and computational analysis of a sea urchin gene," Science, vol. 279, pp. 1896-1902, 1998.

[22] S. Kalir, S. Mangan, and U. Alon, "A coherent feed-forward loop with a SUM input function prolongs flagella expression in Escherichia coli," Molecular Syst. Biol. no. 2005.0006, Mar. 2005 [Online]. Available: $\mathrm{http}: / / \mathrm{www}$.pubmedcentral.nih.gov/articlerender.fcgi?artid=1681456

[23] Z. Schuss, Theory and Applications of Stochastic Differential Equations. New York: Wiley, 1980.

[24] Y. Sun and J. Cao, "Adaptive synchronization between two different noise-perturbed chaotic systems with fully unknown parameters," Physica A, vol. 376, pp. 253-265, 2007. 
[25] W. Lin and W. He, "Complete synchronization of the noise-perturbed Chua's circuits," Chaos, vol. 15, p. 023705, 2005.

[26] S. Huang, "Gene expression profilling, genetic networks, and cellular states: An integrating concept for tumorigenesis and drug discovery," J. Molecular Med., vol. 77, pp. 469-480, 1999.

[27] F. Yang, Z. Wang, and Y. S. Hung, "Robust Kalman filtering for discrete time-varying uncertain systems with multiplicative noises," IEEE Trans. Automat. Control, vol. 47, no. 7, pp. 1179-1183, Jul. 2002.

[28] Z. Wang, F. Yang, D. W. C. Ho, and X. Liu, "Robust finite-horizon filtering for stochastic systems with missing measurements," IEEE Signal Processing Lett., vol. 12, no. 6, pp. 437-440, 2005.

[29] W. Yu, J. Lü, Z. Wang, J. Cao, and Q. Zhou, "Robust $\mathrm{H}_{\infty}$ Control and Uniformly Bounded Control of Genetic Regulatory Network With Stochastic Disturbance," submitted.

[30] J. Zhou, J. Lu, and J. Lü, "Adaptive synchronization of an uncertain complex dynamical network," IEEE Trans. Automat. Control, vol. 51, no. 4, pp. 652-656, Apr. 2006.

[31] W. Yu, G. Chen, and J. Lü, "On pinning synchronization of complex dynamical networks," Automatica, vol. 45, no. 2, pp. 429-435, Feb. 2009.

\section{On the Stability of Positive Linear Switched Systems Under Arbitrary Switching Laws}

Lior Fainshil, Michael Margaliot, and Pavel Chigansky

\begin{abstract}
We consider $\boldsymbol{n}$-dimensional positive linear switched systems. A necessary condition for stability under arbitrary switching is that every matrix in the convex hull of the matrices defining the subsystems is Hurwitz. Several researchers conjectured that for positive linear switched systems this condition is also sufficient. Recently, Gurvits, Shorten, and Mason showed that this conjecture is true for the case $n=2$, but is not true in general. Their results imply that there exists some minimal integer $n_{p}$ such that the conjecture is true for all $n<n_{p}$, but is not true for $n=n_{p}$. We show that $n_{p}=3$.
\end{abstract}

Index Terms-Metzler matrix, positive linear systems, stability under arbitrary switching law, switched systems.

\section{INTRODUCTION}

Consider the linear switched system

$$
\dot{\boldsymbol{x}}(t)=A_{\sigma(t)} \boldsymbol{x}(t), \quad \boldsymbol{x}(0)=x_{0}
$$

where $\boldsymbol{x}(\cdot): \mathbb{R}_{+} \rightarrow \mathbb{R}^{n}, A_{0}, A_{1} \in \mathbb{R}^{n \times n}$, and $\sigma(\cdot): \mathbb{R}_{+} \rightarrow\{0,1\}$ is a piecewise constant function of time, referred to as the switching law. Roughly speaking, this models a system that may switch between the two linear subsystems: $\dot{\boldsymbol{x}}=A_{0} \boldsymbol{x}$ and $\dot{\boldsymbol{x}}=A_{1} \boldsymbol{x}$.

Recall that a function $\alpha:[0, \infty) \rightarrow[0, \infty)$ is said to be of class $\mathcal{K}$ if it is continuous, strictly increasing, and $\alpha(0)=0$. A function $\beta:[0, \infty) \times[0, \infty) \rightarrow[0, \infty)$ is said to be of class $\mathcal{K} \mathcal{L}$ if $\beta(\cdot, t)$ is of class $\mathcal{K}$ for each fixed $t \geq 0$ and $\beta(s, t)$ decreases to 0 as $t \rightarrow \infty$ for

Manuscript received May 18, 2008; revised August 23, 2008 and November 14, 2008. Current version published April 08, 2009. Recommended by Associate Editor D. Angeli.

L. Fainshil and M. Margaliot are with the School of Electrical Engineering-Systems, Tel Aviv University, Tel Aviv 69978, Israel (e-mail: liorfainshil@gmail.com; michaelm@eng.tau.ac.il).

P. Chigansky is with the Department of Statistics, The Hebrew University, Jerusalem 91905, Israel (e-mail: pchiga @ mscc.huji.ac.il).

Color versions of one or more of the figures in this technical note are available online at http://ieeexplore.ieee.org.

Digital Object Identifier 10.1109/TAC.2008.2010974 each fixed $s \geq 0$. We say that (1) is globally uniformly asymptotically stable (GUAS) if there exists a class $\mathcal{K} \mathcal{L}$ function $\beta$ such that for any initial condition $\boldsymbol{x}(0)=\boldsymbol{x}_{0}$ and any switching law $\sigma$ the corresponding solution of (1) satisfies $|\boldsymbol{x}(t)| \leq \beta\left(\left|\boldsymbol{x}_{0}\right|, t\right)$ for all $t \geq 0$. This implies in particular that $\lim _{t \rightarrow \infty} \boldsymbol{x}(t)=\mathbf{0}$.

Denote $A(k):=k A_{0}+(1-k) A_{1}$. By the classic Lie-Trotter product formula [1, Chapter 2]

$$
\lim _{n \rightarrow \infty}\left(\exp \left(k A_{0} / n\right) \exp \left((1-k) A_{1} / n\right)\right)^{n}=\exp (A(k))
$$

for any $k \in[0,1]$. It follows from this that if $A(k)$ is not Hurwitz for some $k \in[0,1]$, then (1) is not GUAS. Thus, from hereon we assume the following.

Assumption 1: Every matrix in

$$
\operatorname{co}\left\{A_{0}, A_{1}\right\}:=\{A(k): k \in[0,1]\}
$$

is Hurwitz.

Assumption 1 is a necessary (but not sufficient) condition for GUAS of (1).

Recently, the problem of establishing conditions that guarantee GUAS of (1) has attracted considerable interest [2]-[7]. A natural idea, first suggested by Pyatnitskiy and his colleagues [8], is to try and characterize the "most unstable" switching law. If the corresponding trajectory is asymptotically stable, then so are all the other trajectories. Thus, the problem can be reduced to analyzing the behavior of this single trajectory. The "most unstable" switching law can be characterized using variational principles (see the survey paper [9]).

Recall that a linear system $\dot{x}=A \boldsymbol{x}$, with $A \in \mathbb{R}^{n \times n}$, is called positive if $\mathbb{R}_{+}^{n}:=\left\{\boldsymbol{x} \in \mathbb{R}^{n} \mid x_{i} \geq 0, i=1, \ldots, n\right\}$ is an invariant set of the dynamics, that is, if $\boldsymbol{x}(0) \in \mathbb{R}_{+}^{n}$ implies that $\boldsymbol{x}(t) \in \mathbb{R}_{+}^{n}$ for all $t \geq 0$. A necessary and sufficient condition for this is that $A$ is a Metzler matrix, that is, all the non-diagonal elements of $A$ are non-negative. Positive linear systems play an important role in system and control theory because in many physical systems the state-variables represent quantities that can never attain negative values (see e.g. [10]).

If both $A_{0}$ and $A_{1}$ are Metzler and $x_{0} \in \mathbb{R}_{+}^{n}$, then we refer to (1) as a positive linear switched system (PLS). PLSs were used for modeling communication systems [11] and formation flying [12] (see also [7]).

Mason and Shorten [13], and independently David Angeli, posed the following conjecture.

Conjecture 1: A PLS that satisfies Assumption 1 is GUAS.

Recently, Gurvits, Shorten, and Mason [14] proved that this conjecture is true for the case $n=2$ (even when the number of subsystems is arbitrary). This result was also proved using the variational approach in [15].

Gurvits, Shorten, and Mason [14] also showed that Conjecture 1 is in general false. As noted in [14], this naturally raises the following question. What is the minimal integer $n_{p}$ for which there exists a PLS that satisfies Assumption 1 but is not GUAS?

In this technical note, we solve this problem by presenting a specific 3 -D system for which Conjecture 1 is false. Since $n_{p}>2$, this proves that $n_{p}=3$.

The remainder of this note is organized as follows. The next section provides a brief review of the results in [14]. Section III presents a specific 3 -D counterexample to Conjecture 1 . The final section concludes.

\section{GuRvits ET AL. COUNTEREXAMPLE}

In this section, we explain the construction in [14]. The first step is an argument that allows transforming a linear switched system with an invariant cone into a PLS. 